\title{
Nonlinear Large Signal Analysis of High Q RF Energy Harvesting Circuits
}

\author{
Christian Merz and Gerald Kupris \\ Deggendorf Institute of Technology \\ Faculty of Electrical Engineering, Media Technology and Computer Science \\ christian.merz@th-deg.de \\ gerald.kupris@th-deg.de
}

\begin{abstract}
The paper focuses on nonlinear analysis of high Q RF energy harvesting circuits. The analysis is made mathematically and by large signal simulation via Keysight Advanced Design System. The mathematical analysis of the nonlinear harvesting circuits is done by using the Galerkin method and the simulations are performed using the harmonic balance method, which is a special version of the Galerkin method. The results are useful for improving harvesting systems, which are used to power wireless and battery-less sensor systems.

Index Terms-Radio Frequency Energy Harvesting, High Q Impedance Matching, Nonlinear Circuit Analysis, Galerkin Method.
\end{abstract}

\section{INTRODUCTION}

The previous work of the authors has focused on high Q impedance matching for harvester networks. It could be shown in a former publication [1] that the use of a high $\mathrm{Q}$ resonator as an inductor in the matching network can lead to an improvement of the harvester efficiency.

This work focuses on the mathematical description of the output voltage in dependence on the input power of an example high $\mathrm{Q}$ harvester network. Because of the nonlinear behavior of the diodes, which are used to rectify the harvested $\mathrm{AC}$ power, the dependence of the output voltage on the input power is also nonlinear. Because of this, the mathematical description of the harvester network leads to a nonlinear differential equation which cannot be solved with algebraic methods. A numerical method has to be used to approximate a solution. A suitable method to find an approximated solution is the so called Galerkin Method, which is used in this work.

\section{METHOD}

The following harvester circuit is analyzed mathematically and by simulation.

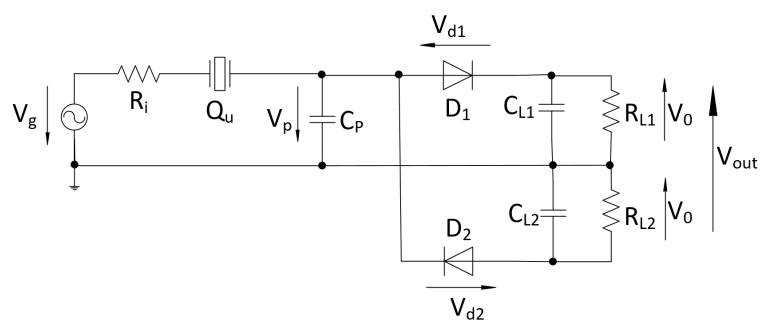

Fig. 1. RF energy harvesting circuit with Delon rectification
The circuit consists of a RF generator block, a high Q matching circuit in L-topology and a Delon rectifier. The RF generator block represents the equivalent circuit of the antenna of the harvester circuit and consists of a RF voltage source $V_{g}$ and a resistance $R_{g}$. The high $\mathrm{Q}$ matching circuit is comprised of a crystal resonator in the series leg and a capacitance in the parallel leg.

A crystal resonator is a resonant circuit with an equivalent circuit (see fig. 2) consisting of a series motional resistance $\left(R_{1}\right)$, a series motional inductance $\left(L_{1}\right)$, a series motional capacitance $\left(C_{1}\right)$ and a shunt static capacitance $\left(C_{0}\right)$. These parameters are also called Butterworth-van-Dyke (BVD) parameters. The three motional devices form a series resonance circuit and the static shunt capacitance forms a parallel resonance circuit together with $L_{1}$ and $C_{1}$.

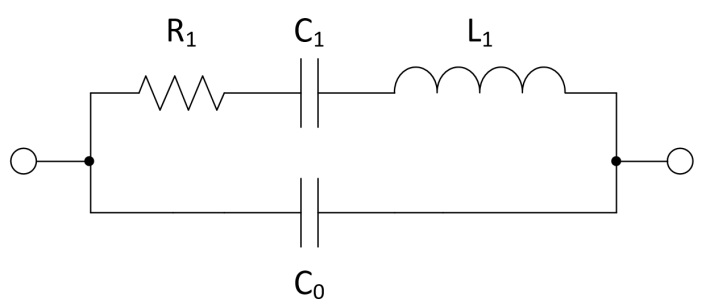

Fig. 2. Equivalent circuit of a resonator

The rectifier includes two HSMS-282 diodes from Avago Technologies and the capacitors $C_{L 1}$ and $C_{L 2}$. The two resistors $R_{L 1}$ and $R_{L 2}$ represent the load of the harvester circuit.

The diodes can be modeled as varistors with a series resistor $R_{j}$ and a junction capacitance $C_{j}$. The following figure shows the equivalent circuit of the diode.

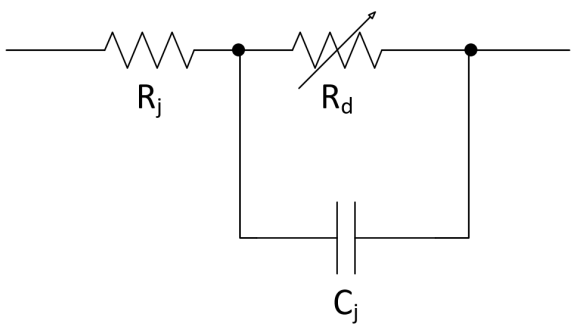

Fig. 3. Equivalent circuit of a diode [2]

The parameters of the HSMS-282 diode are: 
series resistance $R_{j}=6 \Omega$

saturation current $I_{S}\left(\mathrm{~T}=T_{0}\right)=22 \mathrm{nA}$

junction capacitance $C_{j}\left(V_{d}=0 \mathrm{~V}\right)=0.7 \mathrm{pF}$

ideality factor $\mathrm{n}=1.08$

metal-semiconductor energy gap $E_{G}=0.69 \mathrm{eV}$

The current through the diode $I_{D}$ and the diode voltage $V_{D}$ have the following nonlinear relation:

$$
I_{D}=I_{S}(T) \cdot\left[\exp \left(\frac{q \cdot V_{d}}{n \cdot k \cdot T}\right)-1\right]
$$

with:

diode current $I_{D}$

elementary charge $\mathrm{q}=1.06 \cdot 10^{-19} \mathrm{C}$

diode voltage $V_{d}$

Boltzmann constant $\mathrm{k}=1.3806 \cdot 10^{-23} \frac{\mathrm{J}}{\mathrm{K}}$

Using the equivalent circuits of the diode and the resonator, the harvester circuit (see fig. 1) can be designed as illustrated in fig. 4.

To simplify the analysis of the harvester network, the following partial network has firstly to be taken into account:

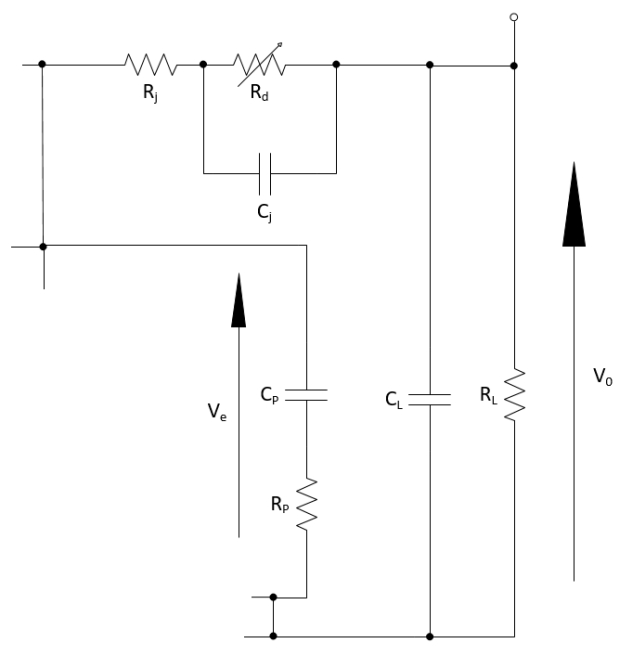

Fig. 5. Partial harvester network

The straightforward analysis of the partial harvester network leads to the following nonlinear differential equation of second order:

$$
\begin{gathered}
I_{S}\left\{\exp \left[\alpha\left(v_{e}(t)+\beta v_{0}(t)+\gamma \frac{d v_{0}(t)}{d t}-v_{0}(t)\right)\right]-1\right\} \\
+C_{j} \frac{d v_{e}(t)}{d t}+\delta \frac{d v_{0}(t)}{d t}+\zeta \frac{d^{2} v_{0}(t)}{d t^{2}}-C_{j} \frac{d v_{0}(t)}{d t} \\
=\frac{v_{0}(t)}{R_{L}}+C_{L} \frac{d v_{0}(t)}{d t}
\end{gathered}
$$

where

$$
\begin{aligned}
\alpha & =\frac{q}{n \cdot k \cdot T} \\
\beta & =\frac{R_{j}}{R_{L}} \\
\gamma & =R_{j} C_{L} \\
\delta & =\frac{R_{j} \cdot C_{j}}{R_{L}} \\
\zeta & =R_{j} C_{j} C_{L}
\end{aligned}
$$

It is not possible to find a closed form of the solution for the differential equation. Because of this, a numerical method has to be used to find an approximated solution. A suitable numerical approximation method is the so called Galerkin method. To apply this method, the differential equation has to be brought into the form

$$
\xi\left[x, y, t, \frac{d}{d t}, \frac{d^{2}}{d t^{2}}\right] \equiv 0
$$

where $\xi$ is a nonlinear operator. The exact solution cannot be determined, but is approximated by an assumed solution

$$
\widetilde{y}(t)=\sum_{k=1}^{N} a_{k} \Phi_{k}(t)
$$

where $\Phi_{k}(t)$ are $\mathrm{N}$ linearly independent functions and $a_{k}$ are $\mathrm{N}$ arbitrary constant coefficients. The assumed solution $\widetilde{y}(t)$ does not in general satisfy the differential equation exactly. Therefore, the expression obtained by substituting equation (4) into equation (3) is no longer equivalent to zero. Instead

$$
\xi\left[x, \widetilde{y}, t, \frac{d}{d t}, \frac{d^{2}}{d t^{2}}\right]=\epsilon(t) \neq 0
$$

where $\epsilon(t)$ is called the residual and is a measure of the deviance of the exact solution and therefore represents the incurred error. This error can be minimized if the residual satisfies the so called Ritz conditions:

$$
\begin{array}{r}
\int_{t_{1}}^{t_{2}} \xi\left[x, \widetilde{y}, t, \frac{d}{d t}, \frac{d^{2}}{d t^{2}}\right] \cdot \Phi_{k}(t) d t=0 \\
k=1,2, \ldots, N
\end{array}
$$

This conditions result in a system of $\mathrm{N}$ algebraic equations with $\mathrm{N}$ variables. To reduce this system of equations to one equation, the ripple of the output voltage has to be neglected, so that the assumed solution changes to

$$
\widetilde{y}=V_{0}
$$

The input forcing function $x$ can be expressed as follows:

$$
x=v_{e}(t)=\hat{V}_{e} \cdot \cos (\omega t)
$$

Here $\hat{V}_{e}$ is the amplitude of the input function and the parameter $\omega$ represents the angular frequency of the RF source.

This simplification leads to only one Ritz condition:

$$
\int_{0}^{T} \xi\left[\hat{V}_{e} \cdot \cos (\omega t), V_{0}, t, \frac{d}{d t}, \frac{d^{2}}{d t^{2}}\right] d t=0
$$




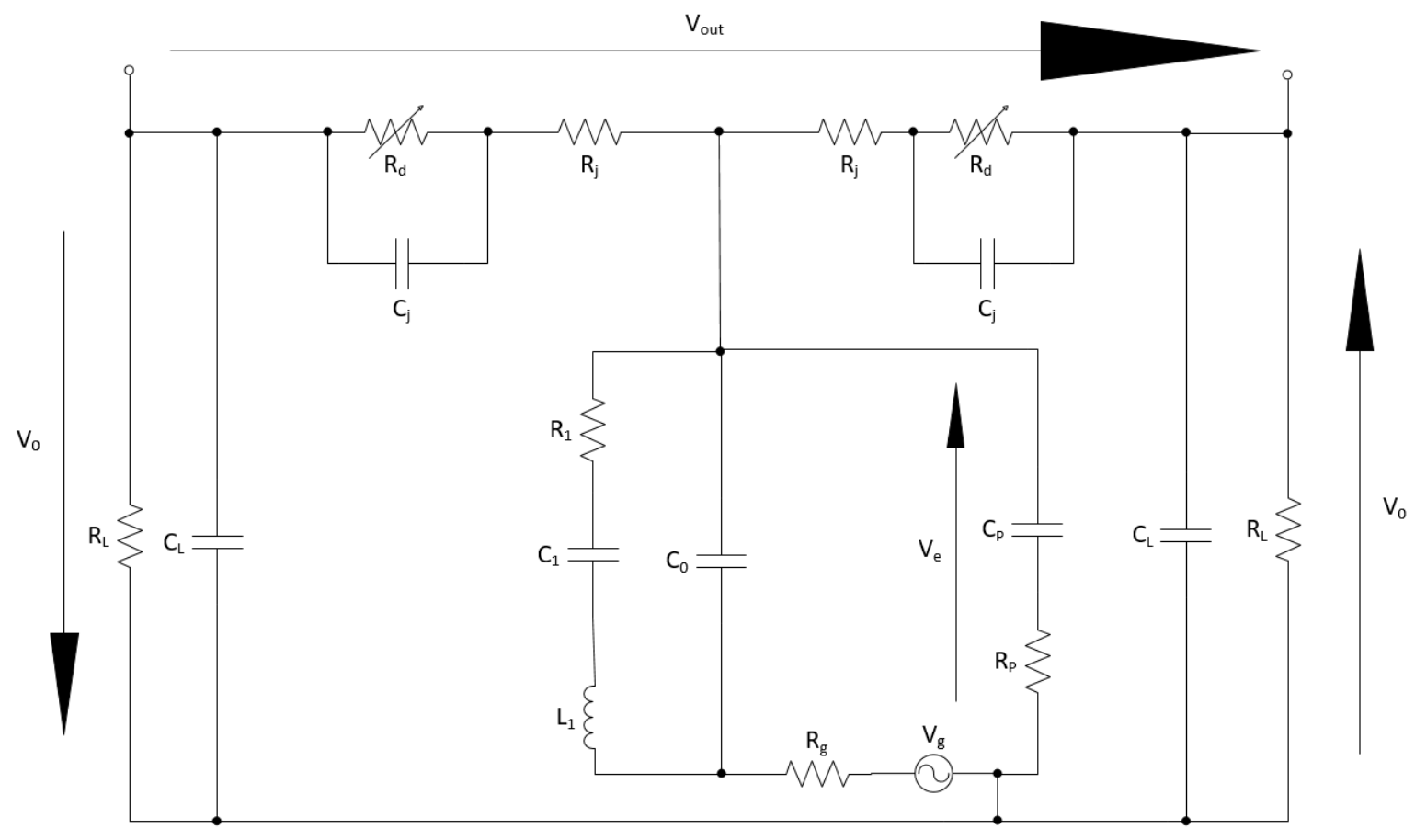

Fig. 4. Harvester circuit with the equivalent circuits of the resonator and the diodes

If the assumed output and input functions, represented by the equations (7) and (8), are inserted into the differential equation (see equation (2)), the residual leads to the following formula:

$$
\begin{array}{r}
\epsilon(t)=I_{S}\left\{\exp \left[\alpha\left(\hat{V}_{e} \cdot \cos (\omega t)+V_{0}(\beta-1)\right)\right]-1\right\} \\
-C_{j} \cdot \hat{V}_{e} \cdot \sin (\omega t)-\frac{V_{0}}{R_{L}}
\end{array}
$$

Carrying out the integration specified in equation (9) and taking equation (10) into account, we get the following nonlinear relation between the input and output value:

$$
\mathbb{I}_{0}\left(\alpha \cdot\left|\hat{V}_{e}\right|\right)=\left(1+\mu \cdot V_{0}\right) \cdot \exp \left(\alpha \cdot \nu \cdot V_{0}\right)
$$

where

$$
\begin{gathered}
\nu=\frac{R_{j}+R_{L}}{R_{L}} \\
\mu=\frac{1}{R_{L} \cdot I_{S}}
\end{gathered}
$$

To get the expression formulated in equation (11), the following formula has been used [3]:

$$
\begin{array}{r}
\mathbb{I}_{0}(z)=\frac{1}{\pi} \int_{0}^{\pi} \exp [ \pm z \cdot \cos (\Theta)] d \Theta \\
=\frac{1}{2 \pi} \int_{0}^{2 \pi} \exp [ \pm z \cdot \cos (\Theta)] d \Theta
\end{array}
$$

$\mathbb{I}_{0}(z)$ is the zero-order modified Bessel function of first kind with argument $\mathrm{z}$. It has to be mentioned that the capacitances $C_{j}$ and $C_{L}$ do not appear in equation (11). This is the consequence of ignoring the ripple component of the output voltage $v_{0}(t)$.
The relation between the absolute value of the input amplitude $\left|\hat{V}_{e}\right|$ and the amplitude of the RF generator source voltage $\hat{V}_{g}$ can be calculated as follows:

$$
\left|\hat{V}_{e}\right|=\left|\frac{\hat{V}_{g} \cdot\left(\frac{1}{j \omega C_{P}}+R_{P}\right)}{R_{g}+Z_{Q}+\frac{1}{j \omega C_{P}}+R_{P}}\right|
$$

The impedance of the crystal resonator $Z_{Q}$ is:

$$
Z_{Q}=\frac{R_{1}+j \omega L_{1}+\frac{1}{j \omega C_{1}}}{\left(R_{1}+j \omega L_{1}+\frac{1}{j \omega C_{1}}\right) \cdot j \omega C_{0}+1}
$$

The relationship between $\hat{V}_{g}$ and the incident power $P_{i n}$, which is fed in at the antenna, can be expressed with the equation

$$
\hat{V}_{g}=\sqrt{8 \cdot R_{g} \cdot P_{i n}}
$$

The output voltage of the harvester system $V_{\text {out }}$ is twice as much as the output voltage of the partial network $V_{0}$ :

$$
V_{\text {out }}=2 \cdot V_{0} \rightarrow V_{0}=\frac{V_{\text {out }}}{2}
$$

$$
\text { III. RESUlts }
$$

The insertion of the formulas (13), (15) and (16) into formula (11) results into the following equation, which describes the nonlinear relation between the output voltage $V_{\text {out }}$ and the input power $P_{\text {in }}$ :

$$
\begin{array}{r}
\mathbb{I}_{0}\left(\alpha\left|\frac{\sqrt{8 \cdot R_{g} \cdot P_{\text {in }}} \cdot\left(\frac{1}{j \omega C_{P}}+R_{P}\right)}{R_{g}+Z_{Q}+\frac{1}{j \omega C_{P}}+R_{P}}\right|\right)= \\
\left(1+\frac{\mu V_{\text {out }}}{2}\right) \cdot \exp \left(\frac{V_{\text {out }}}{2} \cdot \alpha \cdot \nu\right)
\end{array}
$$


The equation (17) has been plotted (see fig. 6) using the following parameters.

- Generator resistance $R_{g}=50 \Omega$

- Equivalent series resistance of the matching capacitor $R_{p}=2.5 \Omega$

- Matching Capacitor $C_{p}=9.3 \mathrm{pF}$

- Series motional resistance $R_{1}=9.78 \Omega$

- Series motional inductance $L_{1}=9.3 \mathrm{mH}$

- Series motional capacitance $C_{1}=14.82 \mathrm{fF}$

- Shunt static capacitance $C_{0}=3.3 \mathrm{pF}$

- Operational frequency $f_{a}=13.562966967 \mathrm{MHz}$

- Load resistance $R_{L}=1 M \Omega$

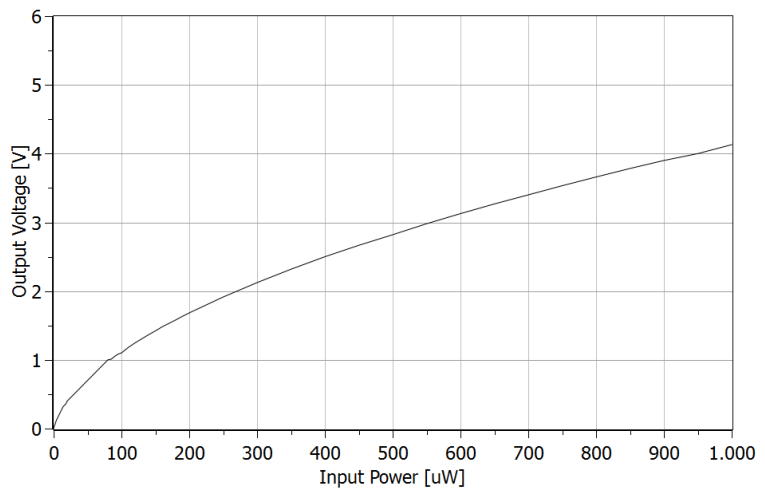

Fig. 6. Output voltage versus input power (calculated)

The output voltage in dependence on the input power has been simulated with Keysight Advanced Design System. The simulation result is shown in fig. 7. The same parameters like in the calculations have been used.

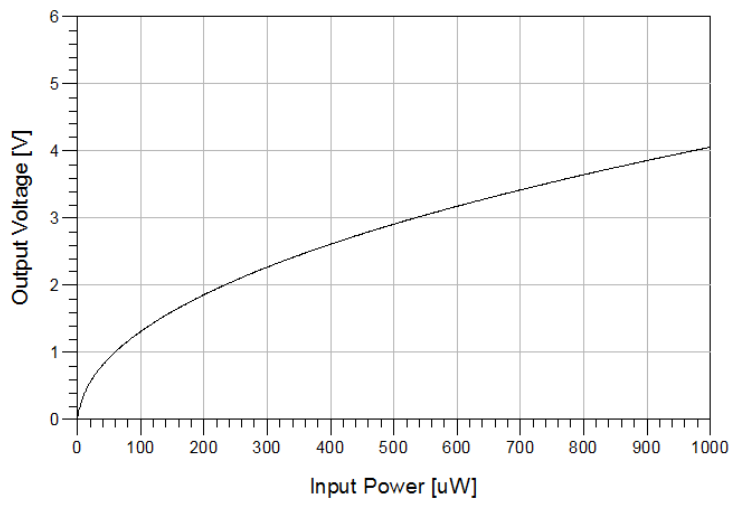

Fig. 7. Output voltage versus input power (simulated)

The simulated and the calculated relation of the output voltage on the input power differs. The reasons for this are the simplifications which are made at the calculations and that not all parameters of the harvester networks has to be taken into account to reduce the complexity of the calculations. The simulation was performed with the so called harmonic balance method, which is a special version of the Galerkin method.

The following fig. shows the spectrum of the output voltage of the partial network $V_{0}$. The first harmonic has a magnitude of $-47.85 \mathrm{dBV}$ and the DC component has a magnitude of $-6.373 \mathrm{dBV}$, which leads to a ripple factor of $0.6 \%$. This shows that the neglection of the harmonics of the output voltage is suitable and does not influence the result significantly.

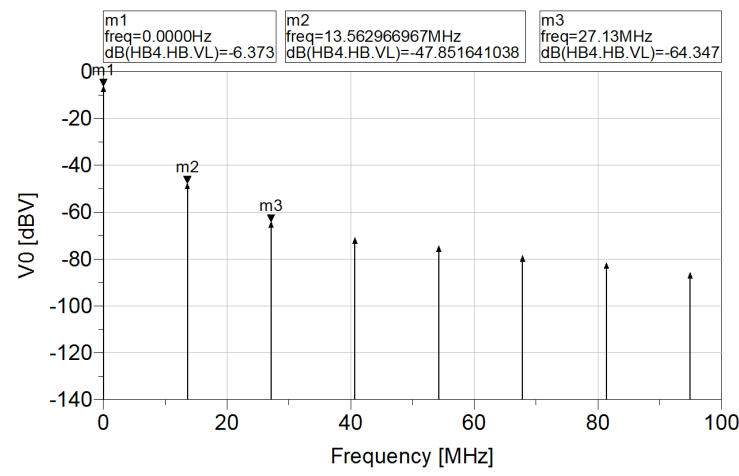

Fig. 8. Spectrum of the output voltage of the partial network $V_{0}$

The value of the matching capacitor $C_{p}$ has been chosen so that the input port voltage reflection coefficient of the harvester network has a minimum between the series resonance frequency $(13.556 \mathrm{MHz})$ and the parallel resonance frequency $(13.586 \mathrm{MHz})$ of the crystal resonator at an input power of $10 \mu \mathrm{W}$.

The following figure shows the input port voltage reflection coefficient versus the frequency at an input power of $10 \mu \mathrm{W}$.

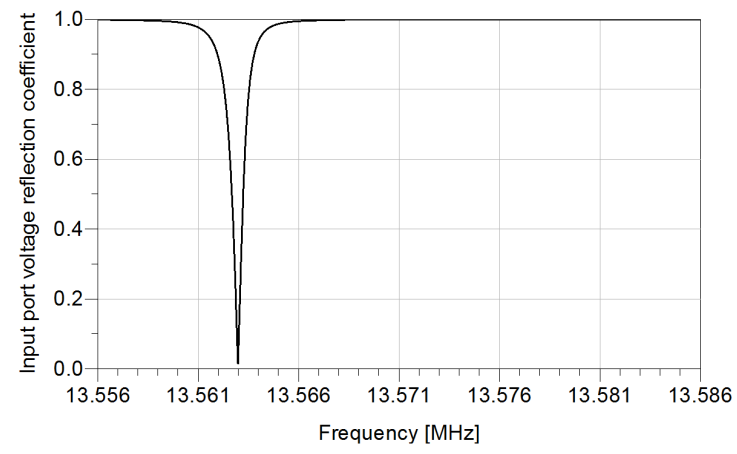

Fig. 9. Simulated voltage reflection coefficient versus the frequency at an input power of $10 \mu \mathrm{W}$

The above figure shows that the input port voltage reflection coefficient has a value of $1.3 \%$ at the operational frequency $f_{a}$.

\section{DISCUSSION}

In this work, a mathematical way has been described to find a relation between the output voltage and the input power of a nonlinear harvester network. The Galerkin method was used to find an approximated solution of the differential equation, which results of the harvester circuit.

\section{REFERENCES}

[1] C. Merz and G. Kupris, High Q Impedance Matching for RF Energy Harvesting Applications, The 3rd IEEE International Symposium on Wireless Systems within the Conference on Intelligent Data Acquisition and Advanced Computing Systems, September 2016, Offenburg, Germany.

[2] Avago Technologies, HSMS-282x Surface Mount RF Schottky Barrier Diodes, Data Sheet, May 2009.

[3] Abramowitz, M. and I. Stegun, Handbook of Mathematical Functions, National Bureau of Standards, June 1964. 